\title{
The effects of freezing on long-term storage of canine erythrocytes
}

\author{
D. Aktaran Bala ${ }^{1}$ M. Özcan² \\ ${ }^{1}$ Istanbul University, Faculty of Veterinary Medicine, Vocational High School, Food Processing Department, \\ Food Technology Programme, 34320 Avcilar/Istanbul/Turkey \\ ${ }^{2}$ Istanbul University, Faculty of Veterinary Medicine, Physiology Department, \\ 34320 Avcilar/Istanbul/Turkey
}

\begin{abstract}
Human medicine studies have so far demonstrated that erythrocytes may be preserved and stored at low temperatures for decades retaining their metabolic and biochemical properties. However, detailed studies regarding this problem are not yet available in veterinary medicine. Therefore, the objective of the current study was to investigate time-dependent effects of long-term frozen storage of canine red blood cells.

Twelve healthy adult dogs meeting the criteria for blood transfusion were used in the study. Whole blood samples $(450 \pm 45 \mathrm{ml})$ collected from each dog were centrifuged by a cryogenic microcentrifuge and packed RBC suspensions were obtained. The samples were prewashed three times in $0.9 \% \mathrm{NaCl}$ solution and were allocated into three groups to be evaluated at three different time points (day 0 and month 4 and 6). The samples to be frozen were subjected to glycerolization and then stored at $-80^{\circ} \mathrm{C}$ for 4 and 6-month periods. At the end of this period the packed RBC samples were thawed, centrifuged and then washed in a consecutive series of dextrose solutions. 2,3-Diphosphoglycerate (2,3-DPG), Adenosin triphosphate (ATP), supernatant hemoglobin (SupHb), sodium $\left(\mathrm{Na}^{+}\right)$and potassium $\left(\mathrm{K}^{+}\right)$levels, residual glycerol concentrations and hemograms were evaluated and compared. Sterility tests were performed on all samples for bacterial contamination. A statistically significant decrease was noted in potassium levels, which was the natural outcome of deglycerolization process. No significant change was observed in terms of other parameters due based on different time points. In conclusion, long-term frozen storage had no negative effect on the quality parameters of canine erythrocytes.
\end{abstract}

Key words: canine, cryopreservation, erythrocyte, glycerol, storage

\section{Introduction}

Novel diagnostic and therapeutic approaches have appeared in veterinary medicine with advance of technology. Likewise, transfusion medicine has gained importance in response to increased demands (Kim et al. 2004, Lucas et al. 2004). Developments in blood storage techniques are important scientific achievement for the maintenance of Blood Banks in terms of the availability of blood products for therapeutic pur- 
poses (Holovati et al. 2009). Blood transfusions and storage methods of blood samples are complementary procedures. (Stokol and Parry 1995, Lucas et al. 2004).

Each component of blood has different and unique function in the organism. Selection of the proper component varies depending on the type of the disease and the requirements of the patient (Battaglia et al. 2000, Sen and Khetarpal 2013). Blood samples collected from the donor animals should either be engaged for an instant use or be separated into the components and stored in most efficient way (Lucas et al. 2004).

With the advances in veterinary transfusion medicine, researchers have discovered the contraindications of the usage of Whole Blood and thus verged to use the blood components, instead, for the treatment of miscellaneous diseases in order to minimize the risks (Kim et al. 2004). The next step was to improve blood storage techniques in an attempt to compensate growing demands for blood transfusion, and therefore, new studies were designed investigating long-term cryopreservation of RBCs (Kim et al. 2004, Kim et al. 2007, Pallota et al. 2012). The aim was to prolong the shelf life of blood products (Pallota et al. 2012) and prevent economic losses.

Studies on cryopreservation of RBCs in human medicine prompted similar studies in veterinary transfusion medicine (Kim et al. 2007), however, research with respect to the determination of alterations or lesions associated with long-term preservation and frozen storage of canine RBCs are far from providing sufficient data. Further studies are still required to investigate the diversity of currently available cryopreservative agents, to improve washing procedures and to determine the storage-induced changes in blood products. Therefore, in the present study, we aimed to determine the time-dependent effects of long-term frozen storage of canine RBCs.

\section{Materials and Method}

Twelve healthy canine donors, meeting the criteria for blood transfusion, were used in the study. Blood samples of $450 \pm 45 \mathrm{ml}$ were obtained from each dog, centrifuged for $5 \mathrm{~min}$ at $4200 \mathrm{x} \mathrm{g}$ and $22^{\circ} \mathrm{C}$ and then packed RBCs were obtained. Samples were prewashed three times in $0.9 \% \mathrm{NaCl}$ solution, each time with the addition of 1.5 fold of the initial samples (AABB Technical Manual 2005d p. 808) by a closed system device (Terumo TSCD-II). Then the samples were divided into three groups to be evaluated on day 0 , and at month 4 and month 6 . Samples to be frozen were subjected to three-stage glycerolization (Glycer- olyte 57, Fenwall) and stored at $-80^{\circ} \mathrm{C}$ for 4 and 6-month periods (AABB Technical Manual 2005d p. 809). At the end of this period RBC units were thawed at $36-38^{\circ} \mathrm{C}$ in a water bath with manual stirrer and then washed via a series of centrifugation steps in $12 \%, 1.6 \%$ and $0.9 \% \mathrm{NaCl}+0.2$ dextrose solutions for the deglycerolization process (Wagner et al. 2000). Quality paramaters were assessed for the samples collected on day 0 and for the samples thawed and deglycerolized.

ATP content was measured by luminometric assay (Berthold Luminometer) with a commercially available kit (PROMEGA G7570 Cell Titer Glo Cell Viability Assay).

2, 3 DPG concentration was assessed by spectrophotometry with commercial kits (Roche Diagnostics) (Lecak et al. 2004).

Extracellular $\mathrm{Na}^{+}$and $\mathrm{K}^{+}$levels were determined by flame photometry (JENWAY) (Valeri et al. 2000).

Hematologic parameters (RBC, Hb, Hct, RDW, $\mathrm{MCV}, \mathrm{MCHC}$, and $\mathrm{MCH}$ ) were assessed by an automatic blood cell counter (Abacus Junior Vet).

Sup. $\mathrm{Hb}$ level was estimated by spectrophotometric method.

Residual glycerol concentration in final supernatant samples was estimated by refractometry (Atago surNh). For this purpose, Wong (2009)'s method has been modified and used. Because the supernatant $\mathrm{Hb}$ concentration is also effective on the refractive index (RI), the mathematical relationship between $\mathrm{Hb}$ and glycerol concentration has been defined by using dilution series and calibration curves. Therefore, dilution series of $\mathrm{Hb}$ and glycerol solutions have been prepared using the final rinsing buffer. RI measurements of the $\mathrm{Hb}$ and glycerol dilution series have been performed by using refractometer and the values plotted against the $\mathrm{Hb}$ and glycerol concentrations (Fig. 1A,B). Finally, residual glycerol formula has been constructed by combining the equations obtained from the calibration curves (Equation 1).

\section{Equation 1}

Equation from Fig. 1A: $y=27.586 x+16$ which means; $\mathrm{RI}=27.586 * \mathrm{Hb}+16$

Equation from Fig. 1B: $\mathrm{y}=0.8 \mathrm{x}+16$ which means; $\mathrm{RI}=0.8 *$ Glycerol +16

$\mathrm{RI}=\mathrm{a} *$ Glycerol $+\mathrm{b} * \mathrm{Hb}+\mathrm{c}$

$\mathrm{RI}=(0.8 *$ Glycerol $)+(27.586 * \mathrm{Hb})+16$

Glycerol $=(\mathrm{RI}-16-(27.586 * \mathrm{Hb})) / 0.8$

$\mathrm{a} / \mathrm{b}$ : Slope of calibration curves of Glycerol/Hb dilutions,

Sterility tests were carried out by using blood culture bottles (Oxoid) against bacterial contamination. 
(A)

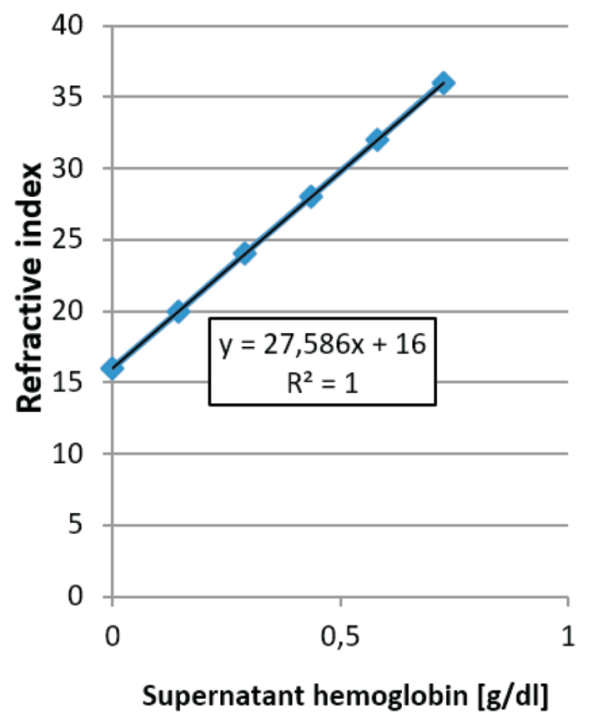

(B)

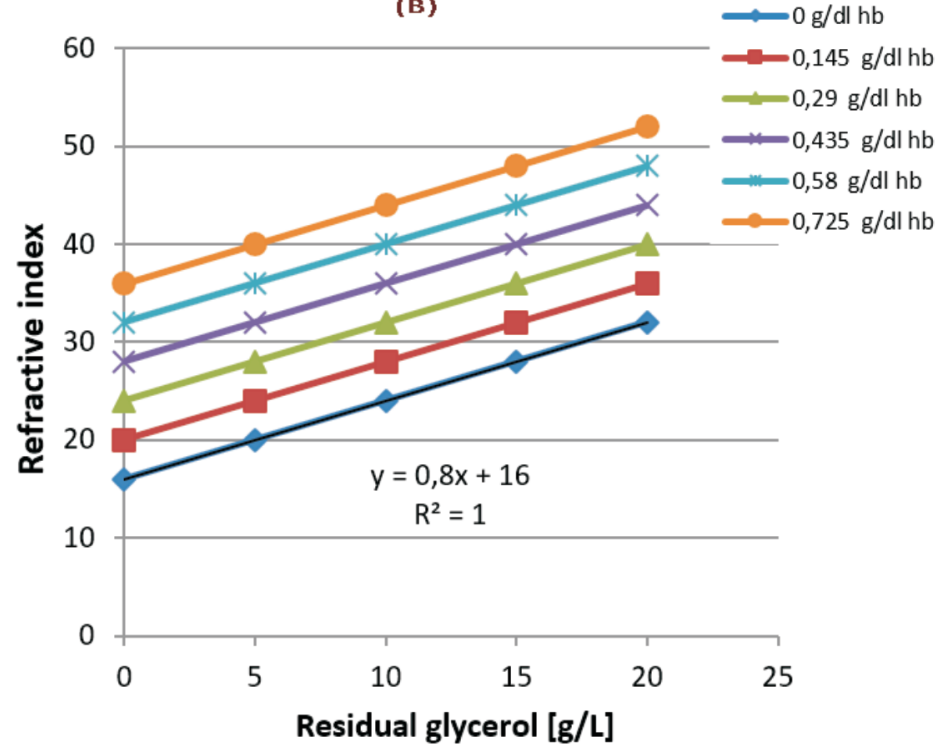

Fig. 1. A, B. Calibration curves for supernatant $\mathrm{Hb}$ and residual glycerol. Since both the supernatant Hb and residual glycerol concentrations are effective on the refractive index (RI), the mathematical relationship between Hb and glycerol concentrations has been defined by using dilution series and calibration curves. For this purpose, dilution series of Hb and glycerol solutions have been prepared using the final rinsing buffer. RI measurements of the $\mathrm{Hb}$ and glycerol dilution series have been performed by using refractometer and the values plotted against the concentrations (Fig. $1 \mathrm{~A}, \mathrm{~B}$ ). Finally, residual glycerol formula has been constituted by combining the equations obtained from the calibration curves (Equation 1).

\section{Resultss}

The cryopreservation procedure was found to be effective in terms of ATP, DPG, $\mathrm{Na}^{+}$, RDW, MCV, and Sup. $\mathrm{Hb}$ levels. A statistically significant decrease was found in terms of the mean values with respect to these parameters at 4 and 6- month periods compared to those on day 0 . No statistically significant difference was found between these two months. Mean $\mathrm{K}^{+}$ values decreased both in comparison with the day 0 and between the two different month intervals and this decrease was found to be statistically significant. An increase was monitored in Hct values only at month 6 although the changes in $\mathrm{RBC}, \mathrm{Hb}, \mathrm{MCH}$ and $\mathrm{MCHC}$ levels were of no statistical significance. Residual glycerol values did not differ between 4 and 6-month time points. (Table 1). No bacterial contamination was evidenced on the basis of sterility tests.

\section{Discussion}

The decreases in ATP and 2, 3 DPG concentrations after 4 months, which were found to be statistically significant $(\mathrm{p}<0.001)$, continued also in the storage period (between $4^{\text {th }}$ and $6^{\text {th }}$ month), however were not statistically significant. It is well known that while glucose concentration decreases due to ultra-low temperature during storage, $\mathrm{pH}$ value decreases as well as a result of an increase in lactic and pyruvic acid levels.
ATP and 2, 3 DPG concentrations are reduced accordingly (Hogman and Meryman 1999, Hess and Greenwalt 2002, Hess 2010).

The changes monitored due to the duration of the storage period following the freezing process were found not to be statistically significant (Table 1). The studies conducted on human (Valeri et al. 2000, Lecak et al. 2004) and canine RBCs (Contreras et al. 1979, Kim et al. 2004) revealed similar results (Sen and Khetarpal 2013).

On the basis of these findings it was suggested that canine RBCs were exposed to oxidative stress (Liu et al. 2002, Kim et al. 2004) during freezing process due to the glycerol solution used and thus a concomitant loss of ATP was observed. Furthermore, canine RBCs were found to be more fragile than the RBCs of other mammalian species (Matsuzava and Ikarashy 1979).

During storage, degradation of 2, 3 DPG, which is closely associated with that of ATP, was already anticipated as a result of the cessation of glycolysis and a drop in the $\mathrm{pH}$ value (Hess and Greenwalt 2002, Kurup et al. 2003, Holme 2005, Scott et al. 2005, Kor et al. 2006). This decrease in 2, 3 DPG level (Table 1) was compatible with the findings of the Valeri et al. (2000) and Lecak at al. (2004), in which human RBCs were stored by freezing and this effect was found to be statistically significant in this study.

In the study, intracellular $\mathrm{K}^{+}$level decreased significantly in all groups due to different time periods. The findings were compatible with those of the 


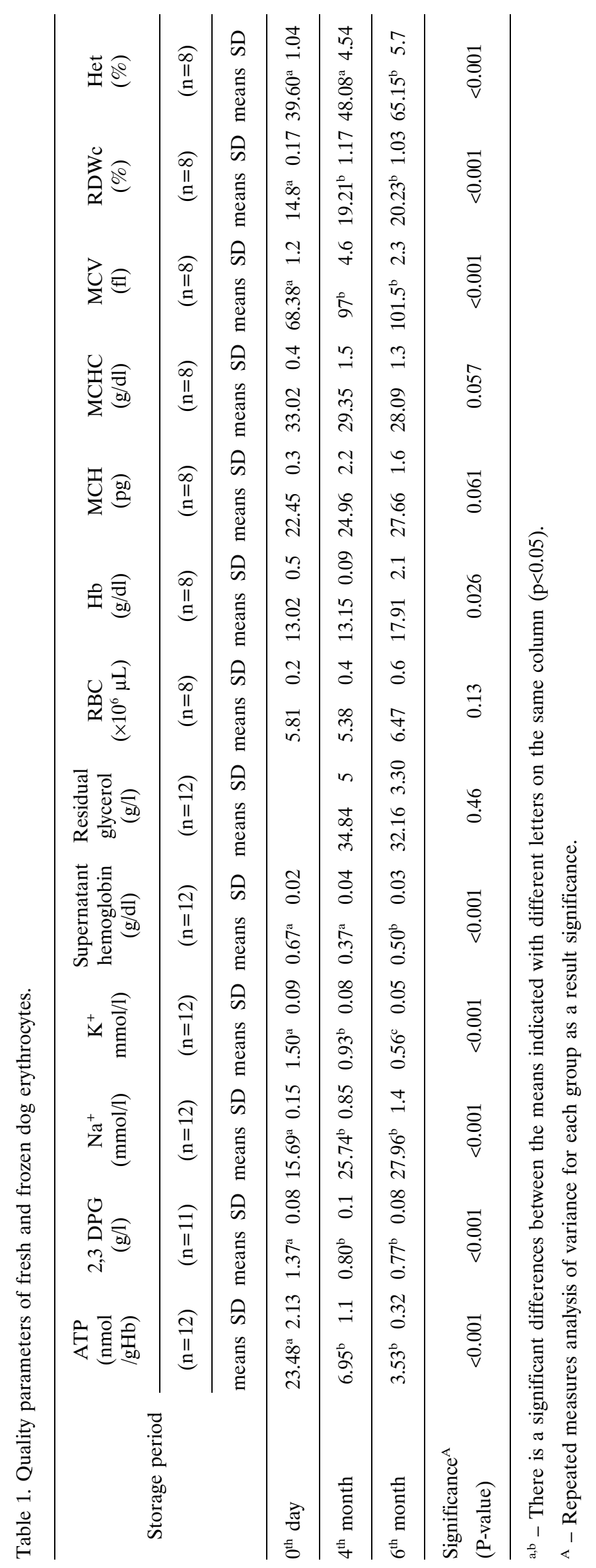


studies conducted on human RBCs (Valeri et al. 2000, Holovati et al. 2008).

Intracellular $\mathrm{K}^{+}$depletion and elevation of $\mathrm{Na}^{+}$ might have resulted from ATP loss. Accordingly, ATP loss due to low temperature was verified to be the consequence of the paralysis of the cellular membrane-associated sodium-potassium pump (Na-K pump) of RBCs (Hess 2010) and degradation of its activity (Hess 2006, Hess 2010, Zubair 2010). Moreover, the function of Na-K pump, which is closely associated with electrolyte balance, requires ATP at certain concentrations (Scott et al. 2005). In the present study, the increase in $\mathrm{Na}^{+}$and the decrease in $\mathrm{K}^{+}$ levels due to the depletion of ATP confirmed our proposition. On the other hand, alterations in electrolyte levels were thought to have resulted from the rinsing series during deglycerolization process since intracellular $\mathrm{K}^{+}$flows out whereas extracellular $\mathrm{Na}^{+}$ flows in through the cellular membrane during freezing and storage processes of RBCs, which eventually serves the electrolyte balance mechanisms (Hogman and Meryman 1999). The results of Holovati et al. (2008), who pointed out the depletion of potassium during deglycerolization was compatible with our findings.

It was a remarkable finding that Sup. Hb concentration of RBCs during prewashing steps on day 0 was estimated to be much more higher than those of frozen RBCs at month 4 and 6 during deglycerolization process (Table 1), which was an evidence of RBC destruction although prewashing procedures make sure that residual substances are removed (Kim et al. 2004).

Sup. Hb concentrations after 4 and 6- month intervals (Table 1) after freezing process were compatible with those of the previous studies (Lecak et al. 2004). Sup. $\mathrm{Hb}$ is defined as the release of hemoglobin in supernatants due to the hemolysis of stored RBCs (Picker et al. 2007). The findings of the present study, similar to those of the former studies, clearly revealed that cryopreserved RBCs underwent oxidative stress and hemolysis due to the sudden temperature change during the thawing process (Kim et al. 2004). Yet, the duration of the freezing period had no effect on this phenomenon. Moreover, residual glycerol concentration did not differ between the 4 and 6-month intervals.

In the study, RBC, $\mathrm{Hb}$ and RDW levels measured during freezing and storage processes (Table 1) revealed similar results to those of the previous studies, in which canine $\mathrm{RBCs}$ were stored at $\left(1-6^{\circ} \mathrm{C}\right)$ low temperatures in additional solutions (Wardrop et al. 1994b). The initial packs of packed RBCs on day 0 were transferred to satellite packs to be evaluated in 4 and 6-month intervals. And thus, increases in RBC,
$\mathrm{Hb}$, Hct levels might have resulted from the difference in cell sedimentation, while the samples were being allocated into groups following glycerolization, during the preparation procedure of $\mathrm{RBC}$ cells and the insufficiency of standardization of the products while being weighed (Wardrop et al. 1994a, Mudge et al. 2004).

MCV mean values at 4 and 6-month intervals raised significantly in comparison with those on day 0 ( $<<0.001$, Table 1$)$. It was suggested that RBCs underwent morphological changes, probably due to the freezing process on the basis of the direct correlation between Hct and MCV levels between the groups, which might be associated with the increase in cellular volume due to the osmotic effects of the supplementary solutions. (Mudge et al. 2004)

Increases in RDW levels, similar to the findings of Pallota et al. (2012), might be considered as an indicator of anisocytosis and cellular deformities at low temperatures (Sollberger et al. 2002).

The statistically insignificant changes in $\mathrm{Hb}$ levels and $\mathrm{MCH}$ and $\mathrm{MCHC}$ mean values were relevant findings (Table 1) and were compatible with those of the studies on cryopreserved human RBCs (Lecak at al. 2004, Holovati et al. 2008).

$\mathrm{MCV}$ mean values increased significantly by 4-month period compared to those on day 0 . The increases after the storage process were found to be statistically insignificant (Table 1), which could be associated with the effect of freezing of RBCs without leukocyte removal by filtration. Parallel to that, it had been reported that substantial increases in $\mathrm{MCV}$ values at hypothermic storage conditions resulted from the cellular membrane damage caused by leukocytes and their waste products (Sollberger et al. 2002).

Since RBCs were processed in a closed system in the study, there was no evidence of bacterial contamination.

\section{Conclusion}

In the present study, freezing process revealed statistically significant changes in the quality parameters of canine RBCs. However, long-term storage of packed RBCs had no significant impact on these parameters, except for potassium levels and hematocrit values. Since alterations in potassium levels were considered to be a natural course of the deglycerolization process, it can be concluded that freezing and long-term frozen storage of canine RBCs has no negative effect on the quality of the product. 


\section{Acknowledgments}

This work was supported by the Research Fund of Istanbul University, Project No. 4322.

\section{References}

Battaglia AM (2000) Small animal transfusion medicine. In: Small animal emergency and critical care. W.B. Saunders, pp 57-71.

Brecher ME (2005) Blood collection,storage and component preparation. American Association of Blood Banks Technical Manual. 15th ed., Bethesda, Maryland, pp 799-818.

Contreras TJ, Lindberg JR, Lowrie GB, Pivacek LE, Austin RM, Vecchione JJ, Valeri CR (1979) Liquid and freeze-preservation of dog red blood cell. Transfusion 19: $279-292$

Hess JR (2006) An update on solutions for red cell storage. Vox Sang 91: 13-19.

Hess JR (2010) Red cell changes during storage. Transfus Apher Sci 43: 51-59.

Hess JR, Greenwalt TG (2002) Storage of Red Blood Cells: New Approaches. Transfus Med Rev 16: 283-295.

Högman CF, Meryman HT (1999) Storage parameters affecting red blood cell survival and function aft er transfusion. Transfus Med Rev 13: 275-296.

Holme S (2005) Current Yssues Related to the Quality of Stored RBCs. Transfus Apher Sci 33: 55-61.

Holovati JL, Hannon JL, Gyongyossy-Issa MI, Acker, JP (2009) Blood preservation Workshop: New and Emerging Trends in Research and clinical practice. Transfus Med Rev 23: 25-41.

Holovati JL, Wong KA, Webster JM, Acker JP (2008) The effects of cryopreservation on red blood cell microvesiculation, phosphatidylserine externalization and CD47 expression. Transfusion 48: 1658-1668.

Kim H, Itamoto K, Tanaka S, Nakaichi M, Sumida S, Taura $Y$ (2007). Influence of pre- freeze treatment and cryo-storage temparature on the post-thaw stability of canine red blood cells cryopreserved in the presence of hydroxyethyl starch. Vet Res Commun 31: 539-543.

Kim H., Tanaka S, Une S, Nakaichi M, Sumida S, Taura Y (2004) A comparative study of the effects of glycerol and hydroxyethyl starch in canine red blood cell cryopreservation. J Vet Med Sci 66: 1543-1547.

Kor DJ, Van Buskirk CM, Gajic O (2009) Red blood cell storage lesion. Bosnian J Basic Med 9: 21-27.

Kurup PA, Arun P, Gayathri NS, Dhanya CR, Indu AR (2003) Modified formulation of CPDA for storage of whole blood, and of SAGM for storage of red blood cells, to maintain the concentration of 2,3-diphosphoglycerate. Vox Sang 854: 253-261.

Lecak J, Scott K, Young C, Hannon J, Acker JP (2004) Evaluation of red blood cell stored at $-80^{\circ} \mathrm{C}$ in excess of 10 years. Transfusion 44: 1306-1313.

Liu J, Christian JA, Critser JK (2002) Canine RBC osmotic tolerance and membrane permeability. Cryobiology 44: 258-268.

Matsuzawa T, Ikarashy Y (1979) Haemolysis of various mammalian erythrocytes in sodium chloride, glucose and phosphate-buffer solutions. Lab Anim Sci 13: 329-331.

Mudge MC, Macdonald MH, Owens SD, Tablin F (2004) Comparison of 4 blood storage methods in a protocol for equine pre-operative autologous donation. Vet Surg 33: 475-486.

Pallota V, D'Amici GM, D'Alessandro A, Rossetti R, Zolla L (2012) Red blood cell processing for cryopreservation: from fresh blood to deglycerolization. Blood Cell Mol Dis 48: 226-232.

Picker SM, Radojska SM, Gathof BS (2007) In vitro quality of red blood cells RBCs collected by multicomponent apheresis compared to manually collected (RBCs) during 49 days of storage. Transfusion 47: 687-696.

Scott KL, Lecak J, Acker JP (2005) Biopreservation of red blood cells: past, present and future. Transfus Med Rev 19: 127-142.

Sen A, Khetarpal A (2013) Comparative study of automated cryopreservation of red blood cells. Med J Armed Forces India 69: 345-350.

Sollberger T, Walter R, Brand B, Contesse J, Meredith DO, Reinhart WH (2002) Influence of prestorage leucocyte depletion and storage time on rheologic properties of erythrocyte concentrates. Vox Sang 82: 191-197.

Valeri CR, Ragno G, Pivacek LE, Cassidy GP, Srey R, Hansson-Wicher M, Leavy ME (2000) An experiment with glycerol - frozen red blood cells stored at $-80^{\circ} \mathrm{C}$ for up to 37 years. Vox Sang 79: 168-174.

Wagner CT, Burnett MB, Livesey SA, Connor J (2000) Red blood cell stabilization reduces the effect of cell density on recovery following cryopreservation. Cryobiology 41: $178-194$.

Wardrop KJ, Owen TJ, Meyers, KM (1994a) Evaluation of an additive solution for preservation of canine red blood cells. J Vet Intern Med 8: 253-257.

Wardrop KJ, Young J, Wilson E (1994b) An in vitro evaluation of storage media for the preservation of canine packed red blood cells. Vet Clin Pathol 23: 83-88.

Wong KA, Nsier N, Acker, JP (2009) Use of supernatant refractive index and supernatant hemoglobin concentration to asses residual glycerol concentration in cryopreserved red blood cells. Clin Chim Acta 408: 83-86.

Zubair AC (2010) Clinical impact of blood storage lesions. Am J Hematol 85: 117-122. 